VOL. 42 (1990) [307-313]

\title{
ON THE CONFORMAL DEFORMATION OF RIEMANNIAN STRUCTURES
}

\author{
YOON-TAE JUNG
}

In this paper, we study a nonlinear partial differential equation on a compact manifold;

$$
\Delta u+r u+H u^{a}=0, \quad u>0,
$$

where $a>1$ is a constant, $r$ is a positive constant, and $H$ is a prescribed smooth function.

Kazdan and Warner showed that if $\lambda_{1}(g)<0$ and $\bar{H}<0$, where $\bar{H}$ is the mean of $H$, then there is a constant $0<r_{0}(H) \leqslant \infty$ such that one can solve this equation for $0<r<r_{0}(H)$, but not for $r>r_{0}(H)$. They also proved that if $r_{0}(H)=\infty$, then $H(x) \leqslant 0(\not \equiv 0)$ for all $x \in M$. They conjectured that this necessary condition might be sufficient.

I show that this conjecture is right; that is, if $H(x) \leqslant 0(\not \equiv 0)$ for all $x \in M$, then $r_{0}(H)=\infty$.

\section{INTRODUCTION}

In this paper, we consider the problem of describing the set of scalar curvature functions associated with Riemannian metrics on a given connected, but not necessarily orientable, compact manifold of dimension greater than or equal to 3 .

We shall call metrics $g$ and $g_{1}$ pointwise conformal if $g_{1}=p(x) g$ for some positive function $p \in C^{\infty}(M)$. Now if a given metric $g$ on $M$, where $\operatorname{dim} M=n \geqslant 3$, has scalar curvature $k \in C^{\infty}(M)$ and we seek $K \in C^{\infty}(M)$ as the scalar curvature of the metric $g_{1}=u^{4 /(n-2)} g$ pointwise conformal to $g$, then $u(>0)$ must satisfy

$$
\frac{4(n-1)}{n-2} \Delta u-k u+K u^{(n+2) /(n-2)}=0,
$$

where $\Delta$ is the Laplacian in the $g$ metric.

In carrying out analysis of (1.1), the sign of the lowest eigenvalue $\lambda_{1}(g)$ of the linear part of (1.1), in other words,

$$
L \phi=-\frac{4(n-1)}{n-2} \Delta \phi+k \phi=\lambda_{1}(g) \phi
$$

\section{Received 6 November 1989}

I would like to thank J.L. Kasdan for reading my paper and discussing the proofs of Lemma 3 and the Theorem in this paper. Partially supported by BSI.

Copyright Clearance Centre, Inc. Serial-fee code: 0004-8729/90 \$A2.00+0.00. 
plays a prominent part because the sign of $\lambda_{1}(g)$ is a conformal invariant. In this paper our results are proved in the case of $\lambda_{1}(g)<0$. For basic existence theorems, we use the method of upper and lower solutions $([2$, p.370-371] or $[5$, Lemma 2.6$])$.

\section{Main RESUlts}

Let $M$ be a compact connected $n$-dimensional manifold, which is not necessarily orientable and possesses a given Riemannian structure $g$. We denote the volume element of this metric by $d V$, the gradient by $\nabla$, and the mean value of a function $f$ on $M$ is written $\bar{f}$, that is,

$$
\bar{f}=\frac{1}{\operatorname{vol}(M)} \int_{M} f d V .
$$

We let $H_{,, p}(M)$ denote the Sobolev space of functions on $M$ whose derivatives through order $s$ are in $L_{p}(M)$. The norm on $H_{s, p}(M)$ will be denoted by \|\|$_{s, p}$. The usual $L_{2}(M)$ inner product will be written $\langle$,$\rangle .$

Lemma 1. Assume $K<0$. Then $K$ is the scalar curvature of some metric pointwise conformal to the given metric $g$ if and only if $\lambda_{1}(g)<0$.

Proof: See Theorem 4.1 in [5].

The above Lemma 1 shows that if $\lambda_{1}(g)<0$, then one can always pointwise conformally deform $g$ to a metric of constant negative scalar curvature $k=-c$, where $c>0$ is a constant. Thus (1.1) reads

$$
\frac{4(n-1)}{n-2} \Delta u+c u=-K u^{(n+2) /(n-2)}, \quad u>0 .
$$

In order to understand (2.1), one must first free it from geometric considerations and consider the equation

$$
-L u=\Delta u+r u=-H u^{a}, \quad u>0,
$$

where $a>1$ and $r>0$ are constants, and $H \in C^{\infty}(M)$. Throughout this paper, we shall assume that all data ( $M$, metric $g$, and curvature $K$, et cetera) are smooth merely for convenience.

Kazdan and Warner showed that if $\lambda_{1}(g)<0$ and $\bar{H}<0$, then there is a constant $0<r_{0}(H) \leqslant \infty$ such that one can solve $(2.2)$ for $0<r_{0}<r_{0}(H)$, but not for $r>r_{0}(H)$ (see Proposition 4.8 in [5]). They also showed that if $r_{0}(H)=\infty$ then $H(x) \leqslant 0$ for all $x \in M$. In fact, they proved that if $H\left(x_{0}\right)>0$ for some $x_{0} \in M$, then $r_{0}(H)<\infty$ (see Proposition 4.10 in [5]). Since $\lambda_{1}(g)<0$, Theorem 2.11 in [5] implies that $H \not \equiv 0$. Kazdan and Warner [5] conjectured that this necessary condition might be sufficient, such as in Theorem 10.5(a) of [4]. Now we shall prove that this necessary condition is also a sufficient condition, that is, if $H(x) \leqslant 0(\not \equiv)$ for all $x \in M$, then $r_{0}(H)=\infty$. 
LEMma 2. (Existence of lower solutions.) Let $H \in L_{p}(M)$ with $p>\operatorname{dim} M$. If $\lambda_{1}<0$, then given any positive continuous function $u$ on $M$, there is a function $u_{-} \in H_{2, p}(M)$ with $0<u_{-}<u$ satisfying $L u_{-} \leqslant H u_{-}^{a}$, that is, $\Delta u_{-}+r u_{-}+H u_{-}^{a} \geqslant 0$.

Proof: See Lemma 2.8 in [5], substituting $-r$ for $h$, where $r$ is a positive constant.

We consider the differential operator

$$
L v=-\Delta v-\alpha H v,
$$

where $\alpha$ is a positive constant and $H \leqslant 0(\not \equiv 0)$. For each $\alpha>0$, if $\lambda_{1}(\alpha)$ is the lowest eigenvalue of (2.3), then

$$
\begin{aligned}
\lambda_{1}(\alpha) & =\min _{v \neq 0} \frac{\|v\|_{2}^{2}+\langle v,-\alpha H v\rangle}{\|v\|_{2}^{2}}, \quad v \in H_{1,2}(M) \\
& =\min \left(\|v\|_{2}^{2}+\langle v,-\alpha H v\rangle\right), \quad\|v\|_{2}=1, v \in H_{1,2}(M) .
\end{aligned}
$$

Note that the eigenfunction is never zero (see Remark 2.4 in [5]). Let $\phi_{\alpha}>0$ be the corresponding eigenfunction of (2.3) with $\left\|\phi_{\alpha}\right\|_{2}=1$, that is,

$$
\Delta \phi_{\alpha}+\alpha H \phi_{\alpha}=-\lambda_{1}(\alpha) \phi_{\alpha}
$$

By integrating (2.4) over $M$, we can see that $\lambda_{1}(\alpha)>0$. Now in order to investigate the behaviour of $\lambda_{1}(\alpha)$ as $\alpha \rightarrow \infty$, we shall prove the following key lemma.

LEMMA 3. Let $M$ be a connected compact manifold without boundary. Let $L$ be as in (2.3) and $\lambda_{1}(\alpha)$ be the corresponding eigenvalue of $L$ for $\alpha>0$. If $H \leqslant 0(\not \equiv 0)$, then $\lambda_{1}(\alpha) \rightarrow \infty$ as $\alpha \rightarrow \infty$.

Proof: For each $\alpha>0$,

$$
\triangle \phi_{\alpha}+\alpha H \phi_{\alpha}=-\lambda_{1}(\alpha) \phi_{\alpha}
$$

where $\phi_{\alpha}>0$ is the corresponding eigenfunction with $\left\|\phi_{\alpha}\right\|_{2}=1$. To prove our conclusion we have several steps.

STEP 1. $\left\{\lambda_{1}(\alpha)\right\}_{\alpha \in N}$ is a strictly increasing sequence. Let $\alpha_{1}<\alpha_{2}$. Since $\Delta \phi_{\alpha_{1}}+$ $\alpha_{1} H \phi_{\alpha_{1}}=-\lambda_{1}\left(\alpha_{1}\right) \phi_{\alpha_{1}}$,

$$
\int_{M} \Delta \phi_{\alpha_{1}} \phi_{\alpha_{2}} d V+\alpha_{1} \int_{M} H \phi_{\alpha_{1}} \phi_{\alpha_{2}} d V=-\lambda_{1}\left(\alpha_{1}\right) \int_{M} \phi_{\alpha_{1}} \phi_{\alpha_{2}} d V .
$$

But the fact that $\partial M=\phi$ implies that

$$
\int_{M} \Delta \phi_{\alpha_{1}} \phi_{\alpha_{2}} d V=\int_{M} \phi_{\alpha_{1}} \Delta \phi_{\alpha_{2}} d V
$$


and also $\phi_{\alpha_{2}}$ satisfies

$$
\Delta \phi_{\alpha_{2}}+\alpha_{2} H \phi_{\alpha_{2}}=-\lambda_{1}\left(\alpha_{2}\right) \phi_{\alpha_{2}}
$$

so we find that

$$
\left(\alpha_{1}-\alpha_{2}\right) \int_{M} H \phi_{\alpha_{1}} \phi_{\alpha_{2}} d V=\left\{\lambda_{1}\left(\alpha_{2}\right)-\lambda_{1}\left(\alpha_{1}\right)\right\} \int_{M} \phi_{\alpha_{1}} \phi_{\alpha_{2}} d V
$$

Since $\phi_{\alpha_{1}}, \phi_{\alpha_{2}}>0$ and $H \leqslant 0(\not \equiv 0)$ on $M$ and $\alpha_{1}<\alpha_{2}, \lambda_{1}\left(\alpha_{1}\right)<\lambda_{1}\left(\alpha_{2}\right)$. Hence $\left\{\lambda_{1}(\alpha)\right\}_{\alpha \in N}$ is a strictly increasing sequence. From (2.5) we find that $\left|\lambda_{1}\left(\alpha_{2}\right)-\lambda_{1}\left(\alpha_{1}\right)\right| \leqslant\|H\|_{\infty}\left|\alpha_{1}-\alpha_{2}\right|$. This means that $\lambda_{1}(\alpha)$ is continuous with respect to $\alpha$.

Suppose $\left\{\lambda_{1}(\alpha)\right\}_{\alpha \in N}$ is bounded. Then there exists $\lambda_{0}$ such that $\lambda_{1}(\alpha)<\lambda_{0}$ and $\lambda_{1}(\alpha) \rightarrow \lambda_{0}$ as $\alpha \rightarrow \infty$.

STEP 2. If $\lambda_{1}(\alpha) \rightarrow \lambda_{0}$ as $\alpha \rightarrow \infty$, then $\alpha \int(-H) \phi_{\alpha}^{2} d V \rightarrow 0$ and $\alpha \int(-H) \phi_{\alpha} d V \rightarrow 0$ as $\alpha \rightarrow \infty$.

The variational characterisation of $\lambda_{1}(\alpha)$ implies that

$$
\begin{aligned}
\lambda_{1}(\alpha+\ell) & =\left\|\nabla \phi_{\alpha+\ell}\right\|_{2}^{2}+(\alpha+\ell) \int(-H) \phi_{\alpha+\ell}^{2} d V \\
& =\left\|\nabla \phi_{\alpha+\ell}\right\|_{2}^{2}+\alpha \int(-H) \phi_{\alpha+\ell}^{2} d V+\ell \int(-H) \phi_{\alpha+\ell}^{2} d V \\
& \geqslant \lambda_{1}(\alpha)+\ell \int(-H) \phi_{\alpha+\ell}^{2} d V .
\end{aligned}
$$

Hence for all $\ell>0$,

$$
\lambda_{1}(\alpha+\ell)-\lambda_{1}(\alpha) \geqslant \ell \int(-H) \phi_{\alpha+\iota}^{2} d V
$$

Since $\lambda_{1}(\alpha) \rightarrow \lambda_{0}$ as $\alpha \rightarrow \infty$, for all $\varepsilon>0$ there exists $\alpha>0$ such that

$$
\left|\lambda_{1}(\alpha)-\lambda_{0}\right|<\frac{\varepsilon}{2}
$$

Then

$$
\begin{aligned}
(\alpha+\ell) \int(-H) \phi_{\alpha+\ell}^{2} d V & =\frac{\alpha+\ell}{\ell} \ell \int(-H) \phi_{\alpha+\ell}^{2} d V \\
& \leqslant \frac{\alpha+\ell}{\ell}\left\{\lambda_{1}(\alpha+\ell)-\lambda_{1}(\alpha)\right\} \\
& \leqslant \frac{\alpha+\ell}{\ell}\left|\lambda_{0}-\lambda_{1}(\alpha)\right|<\varepsilon
\end{aligned}
$$


for sufficiently large $\ell>0$. Hence $\alpha \int(-H) \phi_{\alpha}^{2} d V \rightarrow 0$ as $\alpha \rightarrow \infty$. By the Hölder's inequality,

$$
\begin{aligned}
\left|\alpha \int(-H) \phi_{\alpha} d V\right| & \leqslant \alpha \int|H| \phi_{\alpha} d V \\
& \leqslant \alpha\left(\int H^{2} \phi_{\alpha}^{2} d V\right)\left(\int l^{2} d V\right) \\
& \left.=\operatorname{vol}(M) \cdot\|H\|_{\infty} \alpha \int(-H) \phi_{\alpha}^{2} d V, \quad \text { (note } H \in C^{\infty}(M)\right)
\end{aligned}
$$

so the second assertion in Step 2 follows easily.

STEP 3. Since $\int \phi_{\alpha}^{2} d V=1$ and $\Delta \phi_{\alpha}+\alpha H \phi_{\alpha}=-\lambda_{1}(\alpha) \phi_{\alpha}$,

$$
\int\left|\nabla \phi_{\alpha}\right|^{2} d V=\alpha \int H \phi_{\alpha}^{2} d V+\lambda_{1}(\alpha) \text {. }
$$

But $\left|\alpha \int_{M} H \phi_{\alpha}^{2} d V\right| \rightarrow 0$ as $\alpha \rightarrow \infty$ and $\lambda_{1}(\alpha) \rightarrow \lambda_{0}$, hence $\left\{\int_{M}\left|\nabla \phi_{\alpha}\right|^{2} d V\right\}_{\alpha \in N}$ is bounded. Therefore, $\left\{\phi_{\alpha}\right\}_{\alpha \in N}$ is bounded in $H_{1,2}(M)$. By Kondrakov Theorem ([1], Theorem 2.34), $\left\{\phi_{\alpha}\right\}_{\alpha \in N}$ is compact in $L_{2}(M)$. Thus there exists $\phi_{0} \in L_{2}(M)$ such that $\phi_{n_{\alpha}} \rightarrow \phi_{0}$ strongly, where $\left\{\phi_{n_{\alpha}}\right\}$ is a subsequence of $\left\{\phi_{\alpha}\right\}_{\alpha \in N}$. We may assume that $\phi_{\alpha} \rightarrow \phi_{0}$ in $L_{2}(M)$. Since $\int_{M} \phi_{\alpha}^{2} d V=1$ and $\phi_{\alpha}>0$ on $M, \int_{M} \phi_{0}^{2} d V=1$ and $\phi_{0} \geqslant 0(\not \equiv 0)$. (See [1], Proposition 3.43.) Note that $\int_{M} \phi_{0} d V>0$. But for each $\alpha$,

$$
\int_{M} \Delta \phi_{\alpha} d V+\alpha \int_{M} H \phi_{\alpha} d V=-\lambda_{1}(\alpha) \int_{M} \phi_{\alpha} d V
$$

Since $\lambda_{1}(\alpha) \rightarrow \lambda_{0}$ and

$$
\begin{aligned}
\left|\int_{M} \phi_{\alpha} d V-\int_{M} \phi_{0} d V\right| & \leqslant \int_{M}\left|\phi_{\alpha}-\phi_{0}\right| d V \\
& \leqslant \text { constant } \times\left\|\phi_{\alpha}-\phi_{0}\right\|_{2}^{2} \rightarrow 0 \text { as } \alpha \rightarrow \infty,
\end{aligned}
$$

the right side of (2.6) converges to $-\lambda_{0} \int_{M} \phi_{0} d V \neq 0$. But $\int_{M} \Delta \phi_{\alpha} d V=0$ and $\left|\alpha \int_{M} B \phi_{\alpha} d V\right| \rightarrow 0$ as $\alpha \rightarrow \infty$, so the left side of (2.6) converges to 0 as $\alpha \rightarrow \infty$. Hence we have a contradiction. Thus $\left\{\lambda_{1}(\alpha)\right\}_{\alpha \in N}$ is not bounded, that is, $\lambda_{1}(\alpha) \rightarrow \infty$ as $\alpha \rightarrow \infty$.

Using the previous key Lemma 3, we can prove the following main theorem, that is, the necessary condition $H(x) \leqslant 0(\not \equiv)$ for $r_{0}(H)=\infty$ is also sufficient.

THEOREM. (Existence of upper solutions). If $H(x) \leqslant 0(\not \equiv)$ for all $x \in M$, then (2.2) has a solution for any positive constant $r$, so $r_{0}(H)=\infty$.

Proof: If we show that $L u_{+} \geqslant H u_{+}^{a}$ for some positive function $u_{+}>0$ and any positive constant $r>0$, that is,

$$
\Delta u_{+}+r u_{+}+B u_{+}^{a} \leqslant 0
$$


then Lemma 2 implies that there exists a solution of $(2.2)$, so $r_{0}(H)=\infty$. Let $r$ be any positive constant. If we put $u_{+}=e^{\phi}$, then $\Delta u_{+}=e^{\psi}\left(\Delta \psi+|\nabla \psi|^{2}\right)$. Hence

if and only if

$$
\begin{gathered}
\Delta u_{+}+r u_{+}+B u_{+}^{a} \leqslant 0 \\
\Delta \psi+|\nabla \psi|^{2}+r+H e^{c \psi} \leqslant 0
\end{gathered}
$$

for some function $\psi$ and $c=a-1>0$.

If $L v=-\Delta v-\alpha H v$, then by Lemma 3 the first eigenvalue $\lambda_{1}(\alpha)$ of $L$ converges to $\infty$ as $\alpha \rightarrow \infty$ and $\lambda_{1}(\alpha)$ is continuous with respect to $\alpha$. Hence there is a constant $\alpha>0$ such that $\lambda_{1}(\alpha)=r$. Let $\phi$ be the corresponding eigenfunction, that is,

$$
\triangle \phi+\alpha H \phi=-\lambda_{1}(\alpha) \phi=-r \phi, \quad \phi>0 .
$$

Put $\phi=e^{\tilde{\psi}}$. Then

$$
\Delta \tilde{\psi}+|\nabla \tilde{\psi}|^{2}+r+\alpha H=0
$$

Define $\psi=\tilde{\psi}+\lambda$ for some positive constant $\lambda$. Therefore,

$$
\begin{aligned}
\Delta \psi & +|\nabla \psi|^{2}+r+H e^{c \psi} \\
& =\Delta \tilde{\psi}+|\nabla \tilde{\psi}|^{2}+r+H e^{\tilde{c}+c \lambda} \\
& =-\alpha H+H e^{c \tilde{\psi}+c \lambda} \\
& =B\left(e^{c \tilde{\psi}+c \lambda}-\alpha\right) \leqslant 0
\end{aligned}
$$

for sufficiently large $\lambda$, since $H \leqslant 0(\not \equiv 0)$. This completes our theorem.

\section{REFERENCES}

[1] T. Aubin, Nonlinear analysis on manifolds, Monge-Ampére equations: Grundlehren series 252 (Springer-Verlag, Berlin, Heidelberg and New York, 1982).

[2] R. Courant and D. Hilbert, Methods of mathematical physics: Interscience Vol. II (Wiley, New York, 1962).

[3] J.L. Kazdan and F.W. Warner, 'Existence and conformal deformation of metrics with prescribed Gaussian and scalar curvature', Ann. of Math. 101 (1975), 317-331.

[4] J.L. Kazdan and F.W. Warner, 'Curvature function of compact 2-manifolds', Ann. of Math. 99 (1974), 14-74.

[5] J.L. Kazdan and F.W. Warner, 'Scalar curvature and conformal deformation of Riemannian structure', J. Differential Geom. 10 (1975), 113-134. 
Department of Mathematics Chosun University Dong-Gu

Kwangju 501-759

Korea 